\title{
Promoter specificity mediates the independent regulation of neighboring genes
}

\author{
Christopher Merli, David E. Bergstrom, ${ }^{1}$ Jennifer A. Cygan, ${ }^{2}$ and Ronald K. Blackman ${ }^{3}$ \\ Department of Cell and Structural Biology, University of Illinois, Urbana, Illinois 61801 USA
}

\begin{abstract}
Although enhancers can exert their influence over great distances, their effect is generally limited to a single gene. To discern the mechanism by which this constraint can be mediated, we have studied three neighboring Drosophila genes: decapentaplegic (dpp), SLY1 homologous (Slh) and out at first (oaf). Several dpp enhancers are positioned close to $S I h$ and oaf, and yet these genes are unaffected by the $d p p$ elements. However, when a transposon is located within the oaf gene, the $d p p$ enhancers activate the more distant transposon promoters while still ignoring the closer $S I h$ and oaf start sites. To test whether this promoter specificity accounts for the regulatory autonomy normally found for the three genes, we used in vivo gene targeting to replace the oaf promoter with a $d p p$-compatible one in an otherwise normal chromosome. Strikingly, this chimeric gene is now activated by the $d p p$ enhancers. Thus, the properties of the promoters themselves are sufficient to mediate the autonomous regulation of genes in this region.
\end{abstract}

[Key Words: Promoter specificity; targeted gene replacement; Drosophila; promoter/enhancer interaction; gene regulation]

Received February 21, 1996; revised version accepted April 3, 1996.

Enhancers can function over great distances and interact with almost any kind of promoter. These two features underscore a fundamental concern of gene regulation: How is an enhancer constrained to activate only its appropriate gene while not affecting adjacent ones? With the burgeoning of human gene therapy, this issue of regulatory insulation has become one of practical significance. In particular, it is crucial that regulatory sequences introduced with the therapeutic transgene not affect the expression of the genes near its insertion and vice versa.

For the same reasons, each gene in the cell must maintain its intergenic regulatory autonomy (IRA). To date, experimental evidence has pointed to two distinct models that explain how IRA can be mediated. The first model proposes that aspects of chromatin structure serve to divide the chromosome into independent domains of gene action and that a given enhancer and promoter can interact only if they reside within the same domain (Kitsberg et al. 1991; Laemmli et al. 1992; Dillon and Grosveld 1994; Lewin 1994). It is generally believed that boundary structures, stable nucleoprotein complexes, are formed at the ends of the domain and serve to establish and demarcate the functional unit (Eissenberg and

Present addresses: ${ }^{1}$ The Jackson Laboratory, Bar Harbor, Maine 04609 USA; ${ }^{2}$ Department of Molecular and Cellular Biology, Harvard Universty, Cambridge, Massachusetts 02138 USA.

${ }^{3}$ Corresponding author.
Elgin 1991; Kitsberg et al. 1991; Laemmli et al. 1992; Lewin 1994). Several sequences, which we term domain elements, exhibit properties suggesting that they can establish such boundaries. In vertebrates, these include the locus control region (LCR) of the globin gene cluster (Forrester et al. 1986; Grosveld et al. 1987; Chung et al. 1993) and the A elements of the lysozyme gene (Stief et al. 1989; Bonifer et al. 1990; McKnight et al. 1992). In Drosophila, domain element characteristics are associated with the specialized chromatin structures scs and scs' of the hsp70 locus (Kellum and Schedl 1991; Vasquez and Schedl 1994) and the $s u(H w)$ binding region within the gypsy retrotransposon (Holdridge and Dorsett 1991; Geyer and Corces 1992; Roseman et al. 1993). Most of these domain elements can also act ectopically as insulators to prevent enhancers on one side from acting on promoters on the other side (Stief et al. 1989; Kellum and Schedl 1992; Chung et al. 1993; Cai and Levine 1995; Scott and Geyer 1995). This property is crucial for maintaining IRA.

The second model, termed promoter specificity, states that inherent properties of the promoters and enhancers themselves allow some combinations to interact while others are ineffectual. Such a lock-and-key mechanism is likely to be mediated by the compatibility of the proteins bound at the regulatory elements. Recent work on the ability of an enhancer to utilize alternative promoters differentially within the $A d h$ gene has shown that the discrimination is mediated by differing TFIID-TFIIA 
complexes at the promoters (Hansen and Tiian 1995). To date, little is known regarding the use of this mechanism for mediating intergenic autonomy. Experiments involving transgenic constructs in Drosophila suggest that promoter specificity best explains the independence of the adjacent gooseberry and gooseberry-neuro genes $(\mathrm{Li}$ and Noll 1994), as well as some aspects of Antennapedia Complex expression (Gindhart et al. 1995; Kapoun and Kaufman 1995). However, these experiments were all done using approaches in which the gene's normal chromatin environment had been disrupted.

Our interests in IRA arose from the characterization of the Drosophila decapentaplegic (dpp) gene. Among its many roles, $d p p$ is required for the normal development of imaginal disks (Spencer et al. 1982), the groups of cells destined to become the adult's appendages. Expression of $d p p$ in the disks is controlled by a $30-\mathrm{kb}$ cis-regulatory region, called the disk region, located at the 3 ' end of the gene (Fig. 1) (Masucci et al. 1990; St. Johnston et al. 1990; Blackman et al. 1991). We have identified numerous enhancers in this region, each controlling a distinct subset of the $d p p$ expression pattern in the disks (Blackman et al. 1991; R. Blackman, unpubl.). These enhancers reside 20-35 kb from their preferred $d p p$ promoter (Fig. 1).

The great distance over which the $d p p$ enhancers exert their influence prompted us to examine whether these enhancers also affect other genes in their vicinity. Our initial search for genes near the disk region uncovered the out at first (oaf) gene (Bergstrom et al. 1995). In this paper we report that another gene, named SLY1 homologous (SIh), lies between $d p p$ and oaf. Both oaf and SIh are immediately adjacent to the disk region (Fig. 1) and yet they are unaffected by these $d p p$ enhancers (described herein).

The proximity of $d p p$, Slh, and oaf suggests that mechanisms must be in place to limit the interaction of the $d p p$ enhancers with the adjacent genes. Our ability to elucidate this mechanism has been greatly aided by the insertion of an enhancer trap transposon into the $5^{\prime}$ end of the oaf gene. In this report we first examine the regulation of the promoters in and around the transposon and, second, we use the transposon to mediate in vivo gene targeting (Gloor et al. 1991; Nassif et al. 1994) to replace the oaf promoter with another. This technique has allowed us to examine the effect of the promoter exchange in an otherwise normal chromosome. Using these approaches, we present definitive evidence for the key role of promoter specificity in mediating IRA in the $d p p-o a f$ interval.

\section{Results}

A new gene near dpp

To identify functionally important sequences near the $d p p$ enhancers, we used DNA sequence comparisons between two distantly related species, Drosophila melanogaster and Drosophila virilis (Blackman and Meselson 1986). This comparison uncovered an extensive region of homology, likely to be a new gene, in the 119-121.5 region of the $D$. melanogaster map. Taking into account three probable introns (each $<80$ nucleotides long), the $D$. melanogaster sequence (GenBank accession no. $\mathrm{U} 46124)$ has the potential to encode a protein of 655 amino acids. A BLAST search (Altschul et al. 1990) of GenBank shows that this sequence is strongly related throughout its entire coding region to the $S L Y 1$ gene of yeast, coding sequence F43D9.3 from Caenorhabditis elegans, and the $r$-sly 1 gene of rat with amino acid identities of $32 \%, 44 \%$, and $55 \%$, respectively. The rat and fly genes have $81 \%$ amino acid similarity. Because of this extensive similarity, we have named our gene Slh. Another Drosophila gene, Rop, also shows sequence similarity to $S L Y 1$ (Salzberg et al. 1993), but it has only $18 \%$ identity and its regions of similarity do not encompass the entire coding region. The sequence identity between Rop and SIh is only $11 \%$, suggesting that their functions have diverged greatly.

In yeast, SLY1 is an essential gene involved in vesicle transport between the endoplasmic reticulum and the Golgi apparatus (Dascher et al. 1991; Ossig et al. 1991; Mizuta and Warner 1994). In flies, however, Slh appears to be a nonessential gene because a chromosome with a deletion for most of the Slh-coding region is homozygous viable (Bergstrom et al. 1995).

Although a detailed analysis of $S l h$ is beyond the scope of this paper, certain aspects of its transcriptional regulation are pertinent. The sequence comparisons predicted that Slh RNAs should begin near the oaf gene and

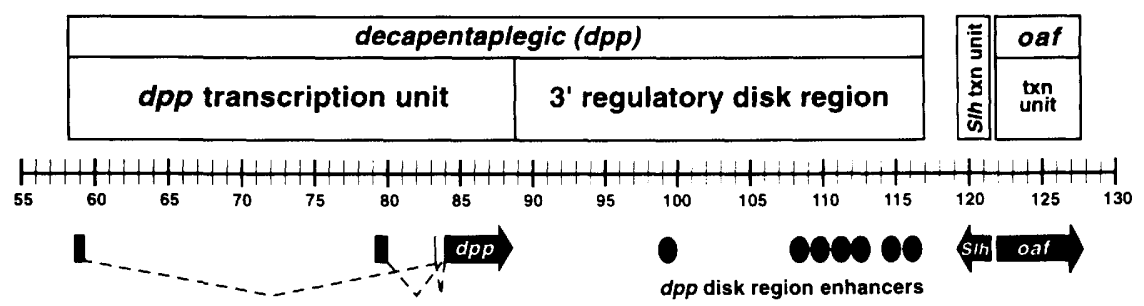
separating their start sites. For $d p p$, three alternative first exons (shown as rectangles) are connected by broken lines to the arrow representing exons 2 and 3 and the intervening intron, which are common to all $d p p$ transcripts. Other minor start sites for $d p p$ RNAs are not shown. Imaginal disk-specific expression of $d p p$ is controlled by enhancers (solid ovals) located within the $3^{\prime}$ disk regulatory region. These enhancers initiate transcription primarily at the start site depicted at map coordinate 79 (St. Johnston et al. 1990 ; R. Blackman, unpubl.).

Figure 1. The molecular organization of $d p p$, Slh, and oaf. The map coordinates (in kilobases| are based on a previous genomic walk (St. Johnston et al. 1990; Bergstrom et al. 1995) and are slightly adjusted as a result of more accurate mapping. For Slh and oaf, the transcription units, including introns, are shown as black arrows. The genes are divergently transcribed with only $300 \mathrm{bp}$ 
be transcribed toward $d p p$. Having found Slh transcripts in embryos (see below), we used 5' rapid amplification of cDNA ends (RACE) (Frohman et al. 1988) on embryonic RNA to map the start of transcription for SIh to a site $300 \mathrm{bp}$ upstream of oaf (see Materials and methods). Thus, oaf and SIh are divergently transcribed with only $0.3 \mathrm{~kb}$ separating their respective start sites (Fig. 1). The two promoters have no apparent sequence homology.

\section{$\mathrm{dpp}, \mathrm{Slh}$, and oaf are independently regulated}

To compare the expression pattern of SIh with $d p p$ and oaf, we performed whole-mount in situ hybridization on embryos and larvae. In embryos, SIh RNA is first evident at stage 14, with signal in the posterior midgut, esophagus, and salivary glands, and remains in these tissues through the remainder of embryogenesis /data not shown). This expression pattern is distinct from that of oaf (Bergstrom et al. 1995) or $d p p$ (St. Johnston and Gelbart 1987) and shows that these closely situated genes are independently regulated.

For this analysis, we used three different singlestranded probes covering the Slh region. Probes extending $\left(5^{\prime} \rightarrow 3^{\prime}\right)$ from 119.5 to 121.0 (containing only Slh antisense sequences) or from 115.1 to 121.0 gave identical results (as described above), whereas a probe extending from 120.8 to 115.1 (containing the sense strand of Slh plus flanking sequences) did not detect expression in any embryonic tissue (data not shown). These results demonstrate that our probes are capable of discriminating localized SIh expression in tissues and suggest that no other embryonic gene is present in the 115-121 interval.

We also used these same probes to examine Slh expression in late third-instar larvae and found no evidence for Slh expression in the anterior structures of these animals. In particular, there was no accumulation of Slh transcripts in the imaginal disks (Fig. 2). At the same time, we also performed hybridizations with probes specific for $d p p$ and oaf transcripts (Fig. 2). The $d p p$ probe yielded its characteristic pattern of a stripe abutting the anterior-posterior compartment boundary in each of the disks (Masucci et al. 1990; Blackman et al. 1991; Raftery et al. 1991). Like Slh, the oaf probe failed to display any reproducible localized pattern within the disks. Unlike Slh, however, the oaf probe always gave a low level of uniform staining in all of the disks. We do not know whether this represents a low level of ubiquitous RNA accumulation or a higher background level specific to the oaf probe. In either case, it is clear that the SIh and oaf transcripts do not accumulate in a $d p p$-like pattern in the imaginal disks. Thus, the $d p p$ enhancers do not activate the promoters of these nearby genes in the wildtype chromosome.

\section{The dpp enhancers can activate heterologous promoters in their vicinity}

Previously, we characterized (Bergstrom et al. 1995) an enhancer trap line, called E32 (Brook et al. 1993), whose $P$ element is inserted within the transcribed, $5^{\prime}$-untrans-

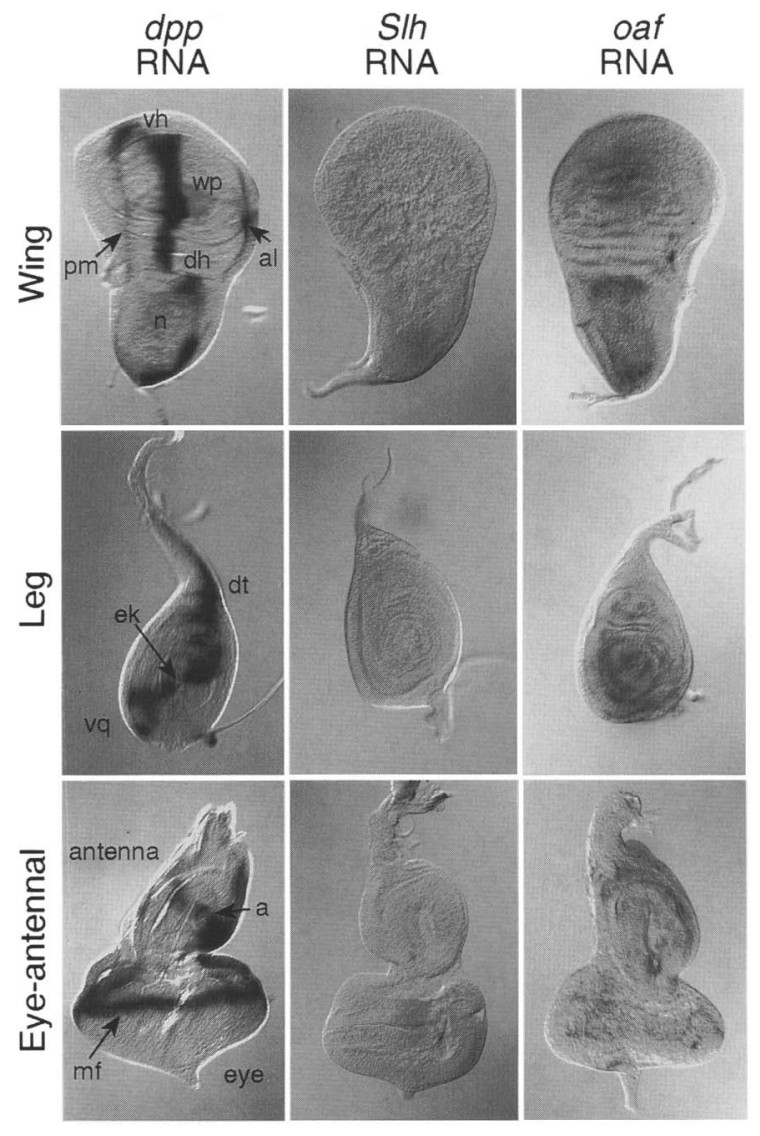

Figure 2. RNA expression in wild-type imaginal disks. Wholemount in situ hybridization with late third-instar disks was performed with riboprobes specific for $d p p$, Slh, or oaf transcripts. For $d p p$ and oaf, cDNA clones were used to prepare the probes. For $S l h$, genomic DNA fragments were used (see text). Data shown in this and subsequent figures were generated from the Slh probe spanning the 119.5-121.0 interval. (Left) In the wing disk, $d p p$ is expressed in a stripe abutting the $\mathrm{A} / \mathrm{P}$ boundary in the ventral hinge (vh), wing pouch (wp), dorsal hinge (dh), and notal $(\mathrm{n})$ regions and in the peripodial membrane $(\mathrm{pm})$. The expression in the presumptive alar lobe (al) region is probably not under the control of the disk region enhancers (Blackman et al. 1991 ; R. Blackman, unpubl.). In the leg disk, $d p p$ transcripts accumulate along the $\mathrm{A} / \mathrm{P}$ boundary from the presumptive dorsal thoracic $(\mathrm{dt})$ region through the end knob (ek, the presumptive tarsal segments) and into the ventral quadrant (vq). (In this and subsequent figures, mesothoracic leg disks only are shown. Pro- and metathoracic leg disks have identical patterns.) In the eye disk, $d p p$ is expressed in the morphogenetic (mf) furrow and in the disk periphery extending from the furrow to the antennal disk. In the antennal disk, $d p p$ RNA accumulates in a stripe passing through the presumptive arista (a). (Center) Slh is not expressed in the imaginal disks. (Right) The oaf probe does not identify any reproducible localized pattern in the disks but instead binds at a low level throughout. We have not determined whether this represents a low level of ubiquitous oaf RNA accumulation or a higher background level specific to this probe.

lated region (UTR) of oaf (Fig. 3). The transposon, inserted after nucleotide +40 , is oriented such that its $l a c Z$ reporter gene and oaf are transcribed in the same 
A

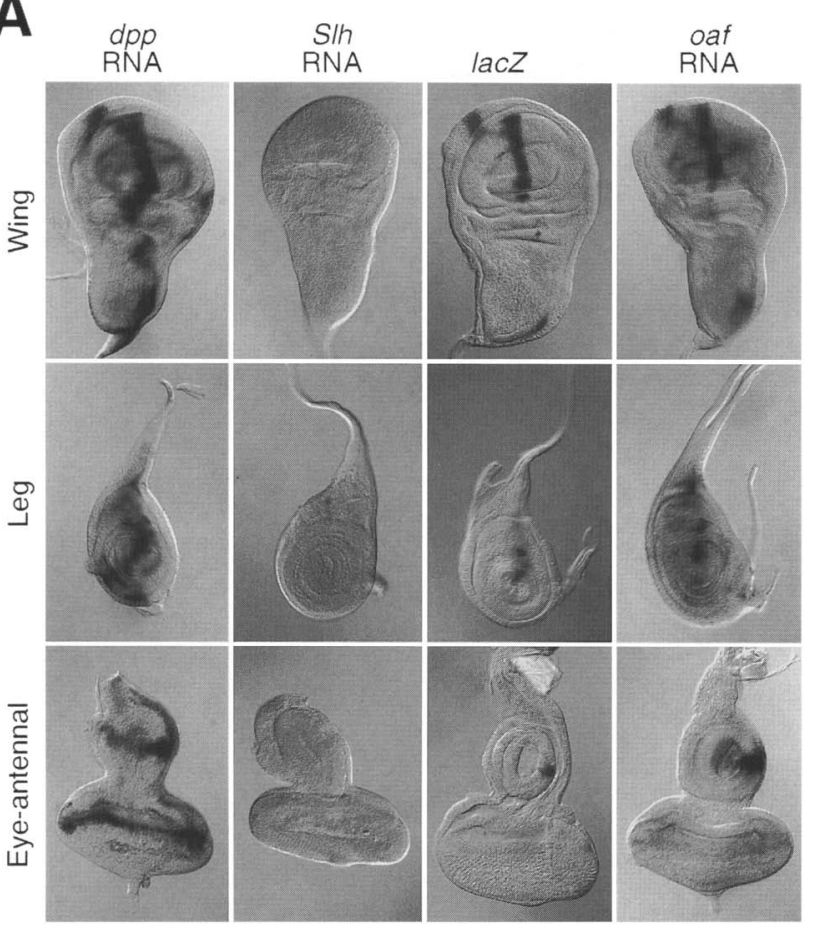

B

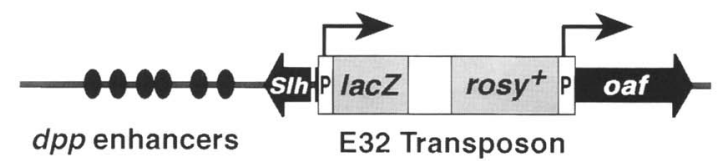

Figure 3. Expression patterns in the $E 32$ line. $|A|$ The expression patterns (from left to right) of $d p p, S l h, l a c Z$, and oaf in flies homozygous for the $E 32$ transposon. The pattern of $d p p$ RNA is indistinguishable from that of wild type, suggesting that $d p p$ expression is not influenced by the insertion of the transposon. No expression is observed for Slh, showing that its pattern is also unchanged from wild type. Histochemical staining for $\beta$-gal (the product of the lac $Z$ gene) shows that this gene's expression is under the regulatory influence of the $d p p$ enhancers. In the wing disk epithelium, the staining reproduces much of the $d p p$ pattern along the $\mathrm{A} / \mathrm{P}$ boundary. Unlike $d p p$, no staining is observed in the upper notal or dorsal hinge regions. The leg disk has expression in the dorsal thoracic and end knob regions with a few clusters of cells staining more intensely. No expression is observed in the ventral quadrant of the disk. In the antennal disk, $\beta$-gal is present in the cells fated to become the arista and antenna segments. There is no staining in the eye disk. The expression pattern of oaf RNA is the same as that of the lacZ gene, showing that its expression in the disks is also controlled by the $d p p$ enhancers. $|B|$ Schematic representation of the chromosome carrying the E32 insertion. The thick arrows indicate the direction and extent of the $S / h$ and oaf transcription units. The E32 transposon (shaded) is inserted after nucleotide +40 of the oaf gene. The darker shaded regions of the transposon correspond to the $l a c Z$ reporter gene and the ry ${ }^{+}$gene, whereas the regions marked $\mathrm{P}$ represent the P-element terminal sequences. The bent arrows indicate the sites of the promoters within the transposon that are activated by the $d p p$ disk enhancers /solid ovals). lacZ transcripts begin around nucleotide +67 of the $P$ element, whereas oaf RNAs begin at a promoter within the right end of the transposon. No transcripts in the disks begin at the endogenous Slh or oaf promoters. direction, with their respective promoters $\sim 100$ bp apart. The 15-kb transposon also contains the Drosophila rosy ${ }^{+}\left(\mathrm{ry}^{+}\right)$gene (used as an eye color marker for transformant selection). Whereas null oaf ${ }^{-}$mutations are ordinarily lethal (Littleton and Bellen 1994; Bergstrom et al. 1995), flies homozygous for the $E 32$ insertion are phenotypically normal and can be maintained as a stock. Functional oaf transcripts in the E32 line are initiated at a cryptic promoter lying near, but within, the right end of the transposon and continue through the oaf-coding region (Fig. 3B; Bergstrom et al. 1995).

Histochemical staining shows that the $\beta$-galactosidase activity ( $\beta$-gal) pattern observed in the E32 line resembles, in general, the normal oaf expression pattern (Bergstrom et al. 1995). There is, however, one critical difference. In $E 32$ larvae, we observe $\beta$-gal in stripes in each of the imaginal disks (Fig. 3), whereas in wild-type larvae there is no such oaf RNA pattern in these tissues (Fig. 2). In the wing disk epithelium, $\beta$-gal is expressed along the anterior-posterior $(\mathrm{A} / \mathrm{P})$ compartment boundary in the cells fated to become the wing, notum, and the ventral wing hinge tissues. In the leg disks, the stripe extends from the presumptive dorsal thoracic region to the central end knob (the presumptive tarsal segments) with several areas staining more strongly. In the eye/antennal disk, only the presumptive arista region produces $\beta$-gal. These patterns constitute a substantial subset of the normal $d p p$ RNA pattern and approximate the pattern directed by the $d p p$ disk enhancers lying in the 110-117 interval (R. Blackman, unpubl.). It is striking that the transposon should respond to the $d p p$ disk enhancers, whereas the SIh and oaf promoters, located in nearly the identical position in the wild-type chromosome, do not.

These results suggest that the $d p p$ enhancers preferentially interact with specific promoters. These enhancers do not activate the promoters of the adjoining genes but can activate a compatible promoter inserted beyond them. An alternative explanation, however, is that the novel action of the dpp enhancers in the $E 32$ chromosome results from an altered chromatin environment. In this view, the insertion of the $E 32$ transposon may disrupt a chromatin-based mechanism that separates the $d p p$ and Slh/oaf genes into separate domains. This perturbation would then allow the $d p p$ enhancers to inappropriately activate any promoter in their vicinity.

To resolve this issue, we have examined more closely the transcription of the $d p p, S l h$, oaf, and lacZ genes in the $E 32$ line. If the transposon does disrupt the domain structure separating $d p p$ from the other genes, then we would expect that each of the genes would interact with the disk enhancers and be transcribed in a $d p p$-like pattern. If promoter specificity mediates IRA in this interval, however, only the ectopic lacZ promoter should be activated, whereas the $S I h$ and oaf promoters will remain quiescent.

\section{The expression pattern of Slh and oaf in the $\mathrm{E} 32$} line supports the promoter specificity model

We first determined the effect of the transposon on the 
expression patterns of the genes in the region (Fig. 3). In situ hybridization for $d p p$ transcripts showed that its expression is normal. Likewise, the pattern of Slh was unaffected by the transposon, showing no expression in the disks. Hybridizations with an oaf cDNA probe, however, uncovered a new pattern. In E32, oaf RNA is present in a pattern virtually identical to that seen for the $\beta$-gal staining. These transcripts are clearly driven by the $d p p$ enhancers and presumably initiate at the cryptic promoter within the right end of the transposon.

We also examined whether the oaf start site or a P-element promoter is used to initiate the lac $Z$ transcripts. In enhancer trap lines such as E32, it is assumed that these RNAs initiate at the promoter of the P-element transposase gene. Two different start sites, at +67 and +87 , have been found for transposase transcripts (Karess and Rubin 1984; R. Blackman, unpubl.). Using $5^{\prime}$ RACE on larval RNA from the E32 line, we found that essentially all transcripts in these animals initiate at or near the +67 start site within the P element /see Materials and methods). Importantly, none of the RACE products began at the normal oaf promoter.

Thus, as diagrammed in Figure 3B, these results indicate that the $d p p$ enhancers ignore the nearer $S I h$ and oaf promoters while activating two transposon promoters, one at essentially the same location and the other $15 \mathrm{~kb}$ away. This use of only some promoters in the region strongly supports the notion that promoter specificity is the overriding feature establishing the regulatory autonomy of these genes.

\section{A direct test of the promoter specificity model}

To test definitively whether promoter specificity is mediating IRA in this interval, we needed to show that the inability of the $d p p$ enhancers to activate SIh and oaf is mediated exclusively through the promoters of these genes. Ideally, these assays should be done with the genes in their normal chromatin environment. We accomplished both of these objectives by replacing the endogenous oaf promoter with a compatible one using $\mathbf{P}$ element-mediated gene replacement (Nassif et al. 1994). This method makes use of the fact that a gap in the chromosome is created when a P element excises. To repair the gap, the cell normally uses the homologous chromosome as the template to replace the missing information (Engels et al. 1990). The Engels and Gloor laboratories have demonstrated, however, that homologous, but altered, sequences located at an ectopic site can also serve as the template /Gloor et al. 1991; Nassif et al. 1994). Thus, using this approach, we could replace the sequences near the $E 32$ insertion site with any that we desired. An important feature of this approach is that the transposon is lost during the process, and the changes are introduced into an otherwise normal chromosome.

We created the replacement template $\mathrm{P}\{\mathrm{ySWAP}\}$ by substituting the -52 to +47 interval of the oaf promoter with the -50 to +65 region of an $h s p 70$ promoter. Because removal of the oaf sequences required the creation of two restriction sites, three additional nucleotide changes were introduced into the replacement template (Fig. 4). We selected this hsp 70 promoter, which lacks the heat shock response element, because it had been activated by the $d p p$ enhancers in other constructs (Blackman et al. 1991; R. Blackman et al., unpubl.). To provide sufficient homology for targeting, the replaced promoter was embedded within a $4.8-\mathrm{kb}$ fragment from the oaf region.

To produce the targeted replacement, we generated flies possessing the ectopic $\mathrm{P}\{\mathrm{ySWAP}\}$ template, the $E 32$ transposon, and the transposase-producing $\mathrm{P}\{\Delta 2-3\}$ element (see Materials and methods). To reduce the chance that the homologous chromosome would be used for repair, we remobilized E32 over a rearranged second chromosome that disrupts pairing in the region near oaf

Figure 4. Comparison of the oaf and oaf ${ }^{\text {SWAP }}$ promoters. The nucleotide numbers given are relative to the start of oaf transcription except for those in the shaded boxes, which are distances relative to the $h s p 70$ start site. In the oaf ${ }^{\text {SWAP }}$ chromosome, the -52 to +47 sequence of the normal chromosome has been replaced with the $h s p 70$ promoter (shaded). Three base pair changes (solid ovals), used to create restriction sites needed for the replacement, are also present in the oaf ${ }^{S W A P}$ chromosome. The two chromosomes differ in length by $16 \mathrm{bp}$, and the start of oaf ${ }^{S W A P}$ transcription is only 2 bases closer to the $d p p$ enhancers

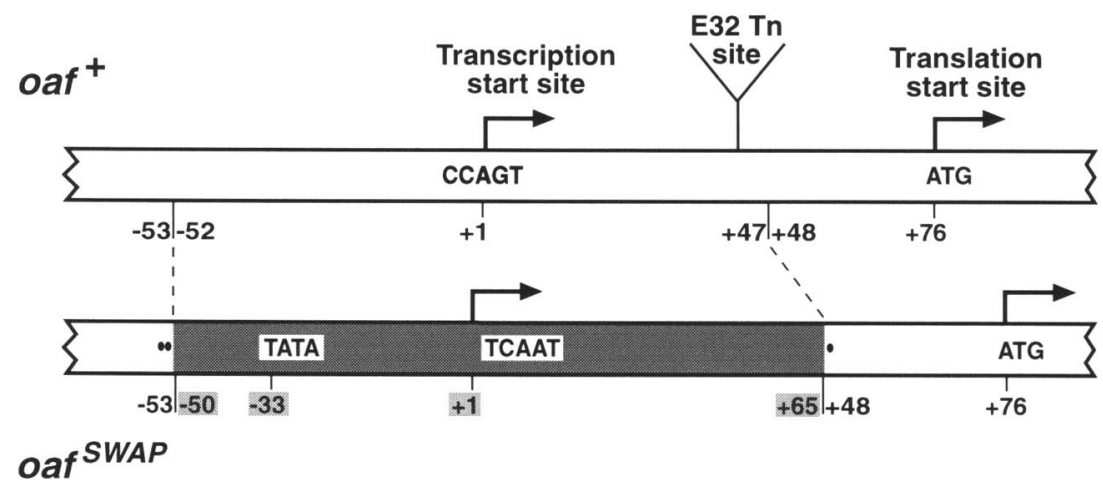
than is the normal oaf start site. The start of translation for oaf ${ }^{S W A P}$ is now 93 bp downstream of the start of transcription. The TATA box of the $O a f^{S W A P}$ sequence is marked; the oaf promoter lacks this motif. Each promoter contains a near-consensus Initiator (Inr) sequence (Cherbas and Cherbas 1993) at the start site. The sequence of the oaf promoter from -60 to +55 is 5 '-dGTCACACCTGTCAGCTGATTTCGAAAAGTCACGTTTCAGTCAGTTTCACCATTTTCTGCCAGTTATTTTTCGTTCGTCGACGCGAGTGGTTCGTTGTGCTTTCTTGTGATTTTAT. The corresponding off ${ }^{\text {SWAP }}$ sequence is 5 '-dGTCAgAtCCGAAAAGAGCGCCGGAGTATAAATAGAGGCGCTTCGTCTACGGAGCGACAATTCAATTCAAACAAGCAAAGTGAACACGTCGCTAAGCGAAAGCTAAGCAAATAAACAAGCGCAGGATCTTAT. Nucleotide changes resulting from the introduced restriction sites are noted by lowercase letters and the $h s p 70$ sequence is underlined. 
(Gelbart 1982). Flies were selected for loss of the $r y^{+}$ gene function associated with the $E 32$ transposon. This phenotype indicates remobilization of the transposon and potential replacement of the oaf promoter. The offspring of $112 \mathrm{ry}^{-}$flies were screened by PCR for the precise replacement of the oaf promoter with the $h s p 70$ sequences. Of these lines, one contained a precise replacement that was verified by both PCR and sequencing. We will refer to this altered gene as $O a f^{S W A P}$. Homozygous oaf ${ }^{S W A P}$ flies appear wild type and can be maintained as a robust stock.

To determine how the altered promoter would affect transcription of the genes in the region, we performed in situ hybridization using $d p p$, Slh, and oaf probes on imaginal disks from homozygous oaf ${ }^{S W A P}$ third-instar larvae (Fig. 5). dpp and Slh are expressed in their normal patterns, demonstrating that they are unaffected by the altered oaf gene. In contrast, oaf ${ }^{S W A P}$ RNA accumulates in a $d p p$-like expression pattern. oaf ${ }^{S W A P}$ expression extends along the $\mathrm{A} / \mathrm{P}$ boundary of the wing disk in the hinge, wing pouch, and notal regions. It differs from the $d p p$ pattern in lacking a portion of the notal expression. In the leg, oaf ${ }^{S W A P}$ expression appears along the $\mathrm{A} / \mathrm{P}$ boundary extending from the dorsal thoracic region to the end knob of the disk. Unlike $d p p$, the expression of oaf ${ }^{S W A P}$ does not continue from the end knob to the periphery on the ventral side of the disk. In the eye/ antennal disk, oaf ${ }^{S W A P}$ accurately reproduces most of the $d p p$ pattern, including the expression in the morphogenetic furrow. As judged by the time needed to produce the signals shown in Figure 5, the levels of $d p p$ and oaf ${ }^{S W A P}$ expression are fairly comparable. These results show clearly that oaf ${ }^{S W A P}$ responds effectively to the $d p p$ enhancers to activate transcription in the disks.

\section{Discussion}

Promoter specificity, not domain boundaries, mediates IRA in this interval

Our results demonstrate conclusively that promoter elements are an essential feature of the mechanism maintaining the regulatory autonomy of the genes within the $d p p-S I h-o a f$ interval. First, in the $E 32$ line, we show that the $d p p$ enhancers ignore the nearer SIh and oaf promoters but still activate two more distant P-element promoters. This finding provides strong support for promoter specificity and adds another important example to the list of genes for which this mechanism is thought to maintain IRA (Li and Noll 1994; Gindhart et al. 1995; Kapoun and Kaufman 1995). The results from each of these studies, however, were obtained under conditions where the assayed promoters and enhancers were not in their native chromatin environments. Thus, any additional aspects of IRA mediated through chromatin structure could not be analyzed in these experiments.

To address this issue, we have tested our model in a completely normal chromatin context. Using a P-element-mediated method for substituting sequences in vivo (Nassif et al. 1994), we replaced the oaf promoter

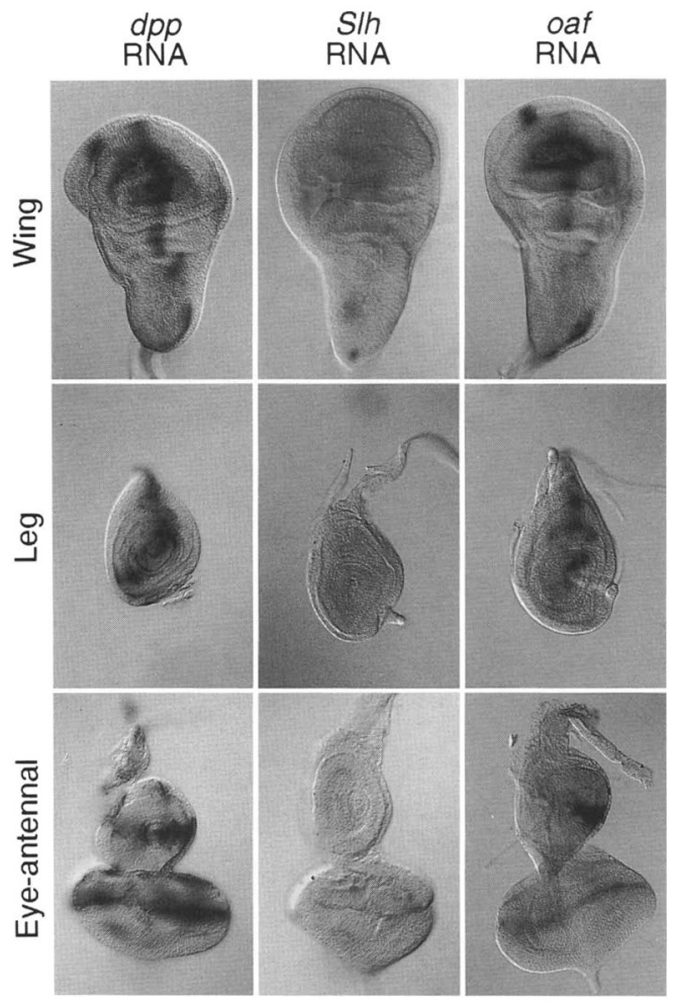

Figure 5. RNA expression patterns in disks from homozygous oaf ${ }^{S W A P}$ larvae. Whole-mount in situ hybridization was performed with riboprobes specific for $d p p$, Slh, or oaf RNA. (Left) The promoter replacement does not affect transcription of the $d p p$ gene. Its expression is indistinguishable from wild type. (Center) Expression of SIh RNA is also not affected by the replacement and shows no localized accumulation. (Right) With its $h s p 70$ promoter, $o a f^{S W A P}$ is activated by the disk enhancers and reproduces nearly all of the $d p p$ pattern. In the wing disk, oaf ${ }^{S W A P}$ RNA appears along most of the A/P boundary and differs from $d p p$ only in lacking staining in part of the notal region. In the leg disk, the entire dorsal thoracic and end knob pattern is seen but there is no reproducible staining in the ventral quadrant. In the antennal disk, oaf ${ }^{S W A P}$ RNA is present in the cells fated to become the arista and antenna. Finally, in the eye disk, Oaf ${ }^{S W A P}$ transcripts accumulate in a discrete stripe in the morphogenetic furrow, exactly the same as $d p p$. Interestingly, this implies that the oaf ${ }^{S W A}$ RNA turns over in the furrow with the same kinetics as the $d p p$ transcripts even though these RNAs have no sequence homology.

with one that is compatible with the $d p p$ enhancers, in effect altering only $100 \mathrm{bp}$ of the chromosome. As predicted by the promoter specificity model, the chimeric oaf ${ }^{S W A P}$ gene responds to the disk region enhancers and is expressed in a pattern essentially identical to that of $d p p$ in the disks. This clearly demonstrates that inherent properties of the promoter are sufficient to determine IRA.

Equally important, our data rule out the presence of an IRA-establishing domain boundary anywhere within the $d p p-S l h-o a f$ interval. Thus, domain boundary-mediated IRA, although important for some genes, may not be as 
widespread as suggested previously (Corces 1995). For the reasons described below, we feel that promoter specificity may be a more common mechanism for IRA.

\section{Domains as mediators of IRA}

There is ample biochemical and cytological evidence that active genes are found within domains of chromatin whose structure is distinct from that of quiescent genes (Elgin 1990; Lewin 1994). It is still unclear, however, whether these biochemically defined units always have a functional role in mediating IRA (Dillon and Grosveld 1994; Schedl and Grosveld 1995). In the reported cases where domain elements establish effectual domains | the globin, lysozyme, heat shock, immunoglobulin, and Bithorax Complex genes (Schedl and Grosveld 1995)], it is interesting to note that nearly all are associated with abundantly expressed loci. It is quite possible that insulated domains are needed principally for cases of exceptionally active genes.

The mechanism by which these domain elements function is unknown and is complicated by the fact that several of the elements have additional regulatory functions. In particular, the globin and lysozyme LCRs contain several gene-specific enhancers /Grosveld et al. 1987; Stief et al. 1989), whereas the scs' element of the heat shock locus contains the promoters for two divergently transcribed, non-heat shock genes /Glover et al. 1995). Further experimentation is needed to discern whether all these domain elements function in similar or distinct manners to mediate IRA.

Given the vast gene number and regulatory complexity of the eukaryotic genome, it is likely that all of the above mechanisms, and more, are needed by the cell to maintain IRA. The list of characterized genes is still too small to know whether any one mechanism will predominate.

\section{Promoter specificity defined}

Implicit in our proposal for promoter specificity is the premise that the $d p p$ enhancers ignore the promoters of the adjacent genes. Our experiments are unable to distinguish whether this results from the physical inability of the enhancers to interact with the promoters or from the active repression of the promoters by gene-specific negative regulatory elements. Although we favor the former explanation, the oaf ${ }^{S W A P}$ results demonstrate that regardless of the mechanism, the inactivity of the oaf gene is dependent on the identity of its promoter. For this reason, we have adopted the term promoter specificity to describe such cases where an enhancer has the inherent ability to interact with some, but not all, promoters in its vicinity. We contrast this mechanism with promoter selectivity, in which an enhancer is capable of activating multiple promoters but opts to activate only one at a given time. This is typically observed in intragenic regulation, where an enhancer selects between alternative promoters of a gene in different tissues or developmental stages [e.g., the Drosophila Adh and actin genes (Corbin and Maniatis 1989; Chung and Keller
1991; Hansen and Tjian 1995)l, but it has also been observed in intergenic situations where an enhancer is shared by separate genes [e.g., the globin loci /Choi and Engel 1988).

\section{Mechanisms mediating promoter specificity}

It is generally assumed that promoter specificity /and selectivity) is mediated by the presence or absence of distinct protein complexes at the promoter and enhancer elements. This mechanism would require the cell to have many functionally different protein complexes capable of binding to promoters and initiating transcription. Given the large number of tissue-specific DNAbinding proteins (Wingender et al. 1996), the varying compositions of the general transcription factor complexes (Parvin et al. 1992; Martinez et al. 1994; Verrijzer et al. 1995), and the heterogeneity of the sequences surrounding promoters (Arkhipova 1995), it seems reasonable that such an extensive array of initiation complexes exists. The important functional differences between promoter sequences can be quite subtle (Das et al. 1995). An example of this was demonstrated by experiments analyzing the TATA elements of the myoglobin and SV40 early gene promoters (Wefald et al. 1990). Although SV40 has the sequence TATTTAT, the myoglobin sequence is TATAAAA. Replacement of the myoglobin TATA with that of SV40 abolished the ability of the myoglobin promoter to respond to a muscle-specific enhancer.

Equally subtle sequence characteristics are probably also responsible for the ability of a promoter to be activated by the $d p p$ disk region enhancers. From our analysis, we know of three compatible promoters: the $d p p$ promoter at map position 79 , which initiates nearly all $d p p$ transcription in the imaginal disks (St. Johnston et al. $1990 ;$ R. Blackman, unpubl.), the oaf ${ }^{\text {SWAP }}$ promoter (mostly that of $h s p 70$ ), and the P-element +67 promoter. Four promoters are not activated by the enhancers: the Slh and oaf promoters, the P-element +87 promoter, and the $d p p$ promoter at map position 83 . Of these, the oaf ${ }^{S W A P}$ and $\mathrm{P}+87$ promoters contain TATA boxes, whereas all but $S I h$ and $P+87$ contain near-consensus Initiator (Inr) elements (Cherbas and Cherbas 1993) at their start sites. The presence of these common promoter motifs in both compatible and noncompatible promoters indicates that neither alone is sufficient to mediate the promoter specificity of the $d p p$ enhancers. Using sequence comparisons, we have been unable to discern any motif that distinguishes between the two classes of promoters.

An alternative mechanism by which promoter specificity could be mediated is one in which the promoter establishes a local chromatin structure that is amenable to certain enhancers but not others. This perturbation (e.g., DNA bending) would have to be quite small in scale, as our experiments showed that the $d p p$ enhancers are capable of differentiating between promoters separated by $<300$ bp in the oaf ${ }^{S W A P}$ chromosome and $110 \mathrm{bp}$ in the E32 chromosome. These distances are less than 
that occupied by two nucleosomes and attest to the local nature of the promoter's effect.

\section{An enhancer can equally activate one or more promoters}

Although our results demonstrate the ability of $d p p$ enhancers to remain indifferent to the SIh and oaf promoters, this does not preclude their ability to activate other promoters in the genome. Presumably, other aspects of physical distance or chromosome structure serve to limit their ability to interact with these distantly located, compatible promoters. When such promoters are brought nearby, however, as in the case of the E32 or oaf ${ }^{S W A P}$ lines, the $d p p$ enhancers show their flexibility by equally activating multiple promoters in the same cell. Strikingly, the presence of the ectopic promoters does not appear to lessen the level of $d p p$ expression in these cells. Although in situ hybridization provides limited quantitative information, our data indicate that the disk region enhancers activate all three promoters in $E 32$ and both promoters in $O a f^{S W A P}$ to a level similar to that of the $d p p$ gene in a normal chromosome. Also, the extreme sensitivity of developing cells to concentrations of $d p p$ protein (Ferguson and Anderson 1992; Wharton et al. 1993; Ingham and Fietz 1995; Zecca et al. 1995) serves as another metric to show that $d p p$ expression has not been altered significantly. If the heterologous promoters had competed away a portion of the $d p p$ enhancer activity, we should have observed $d p p$-like defects. Instead, homozygous $E 32$ and $O a f^{S W A P}$ flies have normal phenotypes. It remains to be seen whether the $d p p$ enhancers function to activate all of the promoters simultaneously or each one alternately, as observed for the LCR activation of human globin genes in transgenic mice (Wijgerde et al. 1995).

\section{Promoter promiscuity}

As our work demonstrates, the fact that an enhancer activates several promoters does not mean that it will interact with all promoters. Some promoters, though, appear to be more promiscuous than others. In the present study, the $h s p 70$ promoter was used because we had shown previously that it could be activated by the $d p p$ disk enhancers (Blackman et al. 1991; R. Blackman, unpubl.). A search of FlyBase reveals that other investigators have used this sequence as a compatible promoter in their constructs as well. Because IRA of the $h s p 70$ locus is maintained by insulating domain boundaries (Kellum and Schedl 1991; Kellum and Schedl 1992; Vasquez and Schedl 1994) and not promoter specificity, it is possible that this promoter needs only to mediate efficient initiation without concern for intergenic enhancer interactions. Although the normal hsp 70 promoter maintains a paused RNA polymerase downstream of its start site (Lis and Wu 1993), the small portion of the promoter that we used in $O a f^{S W A P}$ lacks critical sequences required for generating the paused complex (Lee et al. 1992). Thus, the paused polymerase is unlikely to account for the promoter's facility to be activated.
Most Drosophila enhancer trap transposons make use of the promiscuous nature of the P-element promoters to initiate expression of their reporter gene. Although this has proven very successful, estimates claim that $>50 \%$ of the transposon insertions fail to report all of the regulatory information in their vicinity (Kania et al. 1995). It is likely that some of these failures are caused by the incompatibility of the enhancers with the P-element promoters. Future enhancer trap vectors, using other promiscuous promoters, may identify new genes that to date have evaded detection by the present class of transposons.

\section{In vivo alterations of the Drosophila genome}

We have made use of P-element-mediated targeted gene replacement (Nassif et al. 1994) to introduce our specific modifications into an otherwise normal chromosome. This represents the first such application of the technique for an autosomal location. In our experiments we could not predict a priori that the oaf ${ }^{S W A P}$ chromosome would survive over a deficiency; therefore, we remobilized the transposon over a rearranged chromosome thought to disrupt homologous pairing. Our ability to obtain the targeted replacement in the presence of a wild-type copy of the gene means that essentially any gene with a transposon insertion in or near it is amenable to sequence-specific alteration. With this added flexibility and the large pool of available transposon insertions, P element-mediated sequence replacement should become an indispensable tool for Drosophila molecular genetics.

\section{Materials and methods}

Fly strains

Unless noted, stocks used in this study are described in Lindsley and Zimm (1992). The E32 enhancer trap line (Brook et al. 1993) contains the $\mathrm{P}\left\{1 a c Z^{P Z P T}\right.$ kanR ori $\left.r y^{+t 7.2}=\mathrm{PZ}\right\}$ element inserted after nucleotide +40 of the oaf gene transcription unit (Bergstrom et al. 1995).

\section{Sequence comparisons}

DNA sequencing of $D$. melanogaster and $D$. virilis genomic DNA fragments was performed by the Genetic Engineering Facility at the University of Illinois. Sequences upstream of the oaf gene from both species were compared by use of the MacVector program. A $2.7-\mathrm{kb}$ region of homology was identified and the conceptual translation product compared to the $\mathrm{Na}$ tional Center for Biotechnology Information (NCBI) data bases by use of the BLAST program (Altschul et al. 1990).

\section{5 -End determination of the Slh and E32 transcripts}

The following oligonucleotide primers were used: S1, 5' -dACGCAAGGGCAGTTAGGTCACAAC- $3^{\prime}$; S2, 5'-dGAAGTCATCCTCCAGACTGA-3'; S3，5'-dGTCGGTGGGCAGGCAGAAGT3'; AP20, 5'-dGGCCACGCGTCGACTAGTAC-3'; L1, 5'dGCTGCAAGGCGATTAAGTTG-3'; L2, 5'-dCAUCAUCAUCAUTGGGTAACGCCAGGGTTTTC-3'; P1, 5'-dGTGGGTATCACAAATTTGGG-3'; and P2, 5'-dGGTTCAGGCTCTATCACTTTACT-3'. 
The 5' RACE System from Life Technologies was used to identify the start sites of the transcripts. To map the $\operatorname{Sih} 5^{\prime}$ end, we started with RNA from 8- to 12-hr embryos. cDNA was prepared from $1 \mu \mathrm{g}$ of total RNA with primer Sl, located near the middle of the Slh transcription unit. One-quarter of the cDNA was poly[d(C)]-tailed and subjected to PCR in a $25-\mu]$ volume containing PCR buffer III (Ponce and Micol 1992), 200 $\mu \mathrm{M}$ dNTP, 1 unit of Taq polymerase (GIBCO/BRL), and $0.4 \mu \mathrm{M}$ each of the Anchor Primer supplied with the kit and nested $S I h$ primer S2. Samples were subjected to a thermocycling scheme of $3 \mathrm{~min}$ at $94^{\circ} \mathrm{C} ; 25 \mathrm{cycles}$ of $30 \mathrm{sec}$ at $94^{\circ} \mathrm{C}, 30 \mathrm{sec}$ at $54^{\circ} \mathrm{C}$, and $30 \mathrm{sec}$ at $72^{\circ} \mathrm{C}$; and $10 \mathrm{~min}$ at $72^{\circ} \mathrm{C}$. For the second round of PCR, we used primers S3 and AP20. The former is from SIh and lies nearer the 5' end of the gene, whereas the latter contains the 20 core nucleotides from the Anchor Primer. These primers were used in a $100-\mu$ l reaction containing $4 \mu$ l of round 1 product. The same PCR conditions were used as before except that 30 cycles were performed. This reaction produced a single product (when analyzed by agarose gel electrophoresis) of $\sim 400 \mathrm{bp}$. The DNA was cloned into the pGEM-T vector (Promega), and eight clones sequenced. Seven terminated at the same nucleotide, and one was a single nucleotide shorter.

To map the $5^{\prime}$ end of the transcripts responsible for the $\beta$-galactosidase expression in the $E 32$ line, we used RNA from wandering third-instar larvae. cDNA was primed with random hexamers, tailed, and used in PCR with the Anchor Primer and lacZ primer Ll. Cycling conditions were $2 \mathrm{~min}$ at $94^{\circ} \mathrm{C} ; 30$ cycles of $1 \mathrm{~min}$ at $94^{\circ} \mathrm{C}, 1 \mathrm{~min}$ at $57^{\circ} \mathrm{C}$, and $2 \mathrm{~min}$ at $72^{\circ} \mathrm{C}$; and $10 \mathrm{~min}$ at $72^{\circ} \mathrm{C}$. In controls with plasmid DNA or untailed cDNA, we found that the Anchor Primer inappropriately anneals to a sequence around nucleotide 300 of the P element, resulting in an artifactual product that was observed in later rounds of PCR amplification. From the first reaction, $0.05 \mu \mathrm{l}$ of unpurified product was amplified with nested $l a c Z$ primer $L 2$ and the Universal Amplification Primer supplied with the RACE kit. An appropriately sized fragment along with three smaller products were visible by gel analysis. A third round of PCR was done with the AP20 primer and either of two primers, P1 and P2, whose 5 ' ends are at nucleotide 293 and 379 , respectively, of the $P$ element. Gel analysis showed a single product in the reaction with P1 (260 bp) and two fragments with P2 (370 and $110 \mathrm{bp}$ ). In the latter reaction, the smaller fragment was the product resulting from the Anchor Primer inappropriately priming within the $P$ element. The two largest fragments were cloned and sequenced. Of 23 clones, 21 ended at nucleotide 69 or 70 of the $P$ element. The other two clones also ended within the P element. Similarly sized products were observed in other independently run experiments with different RNA preparations.

\section{Expression patterns in imaginal disks}

Whole-mount RNA in situ hybridization and histochemical staining for $\beta$-galactosidase activity were performed as described in Bergstrom et al. (1995). For these experiments, y $w$ animals served as our wild-type control strain. All RNA probes were generated from DNAs subcloned into pBS II KS. Plasmid pBS-dppB1 contains a 3.2-kb dpp transcript B cDNA originally present in clone KP1 (St. Johnston et al. 1990). Plasmid pBSoafNN9 contains a full-length 3.2 -kb oaf cDNA from clone NN (Bergstrom et al. 1995). A 1.5-kb antisense riboprobe specific for Slh was prepared from genomic clone $\mathrm{pBS} 119 \mathrm{BH}$, covering the 119.5-124.4 interval, which had been linearized at an XhoI site at 121.0. Riboprobes covering the entire insert of clone pBS115CX, spanning the 115.1-121.0 interval, were prepared from each strand.

\section{Construction of the P $\{y S W A P\}$ template}

To create the replacement template, we first subcloned a $1.9-\mathrm{kb}$ XhoI-EcoRI fragment (map coordinates 120.8-122.7) containing the oaf promoter into the pALTER-1 vector from the Altered Sites II in vitro Mutagenesis System (Promega). Following the manufacturer's protocol, we created BglII sites at -57 to -52 and +47 to +52 relative to the start of oaf transcription. The resulting plasmid was cut with $B g l I I$, and the ends filled in with Klenow. The eliminated -52 to +47 sequence was replaced with the -50 to +65 sequence from the $h s p 70$ promoter found in plasmid pHSREM1 (Knipple and Marsella-Herrick 1988). The $1.9-\mathrm{kb} X$ XoI-EcoRI fragment of this chimeric construct was used to replace its wild-type counterpart in a larger subclone extending $2.3 \mathrm{~kb}$ upstream and $2.8 \mathrm{~kb}$ downstream of the oaf start site. A $4.8-\mathrm{kb} \mathrm{XbaI}$ fragment from this plasmid was inserted into the $X b a I$ site of the transformation vector $\mathrm{P}\left\{\mathrm{y}^{+m D i n t}=\mathrm{YC} 4\right\}$ to create the replacement template $\mathrm{P}\left\{\mathrm{y}^{+m D i n t}\right.$ oaf fs. $P-S W A P=\mathrm{ySWAP}\} . \mathrm{P}\{\mathrm{YC} 4\}$ (kindly provided by Pamela Geyer, University of Iowa, Iowa Cityl contains the intronless yellow $(y)$ gene subcloned into the Carnegie 4 vector. $\mathrm{P}\{\mathrm{YC} 4\}$ transformants in a $y$ background have wild-type body and wing pigmentation (Geyer and Corces 1987).

\section{Gene targeting and promoter replacement}

We injected P\{ySWAP $\}$ and helper transposase DNA into $y$ $a c ; r y{ }^{506}$ embryos using standard methods and recovered a transgene insertion, $P\{y S W A P\}$, on the $\mathrm{X}$ chromosome. Females of the genotype $y$ ac $P\{y S W A P\} / y$ ac; $E 32 / C y O$; ry were then crossed to $\operatorname{In}(2 L R) D T D 42, d p p^{d-h o} ;$ ry $P\left\{r y{ }^{+} \Delta 2-3\right\} 99 B$ males to induce excision of the E32 transposon. $\operatorname{In}(2 L R) D T D 42$ is a rearranged second chromosome that is thought to disrupt chromosome pairing near $d p p$ (Gelbart 1982), whereas the $\Delta 2-3 \mathrm{P}$ element serves as an immobile source of $P$ transposase (Robertson et al. 1988). y ac; E32/In(2LR)DTD42, $d p p^{d \cdot h o} ;$ ry/ry $P\left\{r y^{+}\right.$ $\Delta 2-3\}$ 99B males were crossed individually to $d p p^{d-h o}$; ry females. Phenotypically $\mathrm{y}^{-} \mathrm{dpp}^{+} \mathrm{ry}^{-}$males of the genotype y $a c$; $E 32^{*} /$ dpp $^{d \cdot h o}$; ry /where E32* denotes the remobilized transposon chromosome/ were recovered from 112 of the crosses. The loss of the $\mathrm{ry}^{+}$function from the $E 32$ chromosome signals that a remobilization event occurred, possibly leading to the genetargeted replacement of the oaf promoter. The selected males were crossed to establish stocks. These were tested for the promoter replacement by PCR with two different primers inside the $h s p 70$ sequence paired with three different oaf gene primers. The oaf primers were from gene sequences not present in P\{ySWAP\} to ensure that proper PCR products would be amplified only from flies carrying the $h s p 70$ promoter in the native oaf gene. Of the 112 lines, one produced the appropriate PCR products expected of a replacement event. The amplified products were cloned and sequenced to confirm the precise promoter exchange. The chromosome bearing the chimeric gene, which we call oaf ${ }^{S W A P}$, can be maintained as a homozygous stock and has wild-type phenotype.

\section{Acknowledgments}

We are grateful to Pedro Alverez-Ortiz, Richard Shelby and Simi Thomas for technical assistance, Paige Goodlove of the University of Illinois Biotechnology Center for assistance in sequencing and primer design, and Sara Browning and Ellen Omi for help with maintaining the laboratory. We thank Pamela Geyer for supplying fly lines and the YC4 plasmid, Bill Engels for P-element primers and ideas, and Greg Gloor for his advice on the targeted gene replacement protocol. Also, we thank Pamela Geyer, Andy Belmont, and David Rivier for their insightful dis- 
cussions and comments on the manuscript. Finally, R.K.B. especially expresses his gratitude to Joel Eissenberg for his perspicacity, eloquence, and antipodal nature-all of these have made this work and our friendship that much better. This work has been supported by grants to R.K.B. from the National Science Foundation (DCB 90-18618 and MCB 93-17701) and the University of Illinois Research Board. C.M. and D.E.B. were partially funded by National Institutes of Health predoctoral training fellowships.

The publication costs of this article were defrayed in part by payment of page charges. This article must therefore be hereby marked "advertisement" in accordance with 18 USC section 1734 solely to indicate this fact.

\section{Note added in proof}

Using RT-PCR, we have determined that oaf RNA is present in wild-type imaginal disks. Coupled with our in situ hybridization results, this suggests that the oaf promoter is competent for transcription in all disk cells. Thus, the failure of oaf to be activated by the $d p p$ enhancers does not result from the inability of the promoter to function in these cells.

\section{References}

Altschul, S.F., W. Gish, W. Miller, E.W. Myers, and D.J. Lipman. 1990. Basic local alignment search tool. I. Mol. Biol. 215: 403-410.

Arkhipova, I. 1995. Promoter elements in Drosophila melanogaster revealed by sequence analysis. Genetics 139: 13591369.

Bergstrom, D.E., C.A. Merli, J.A. Cygan, R. Shelby, and R.K. Blackman. 1995. Regulatory autonomy and molecular characterization of the Drosophila out at first gene. Genetics 139: $1331-1346$.

Blackman, R. and M. Meselson. 1986. Interspecific nucleotide sequence comparisons used to identify regulatory and structural features of the Drosophila hsp82 gene. I. Mol. Biol. 188: 499-515.

Blackman, R.K., M. Sanicola, L.A. Raftery, T. Gillevet, and W.M. Gelbart. 1991. An extensive 3' cis-regulatory region directs the imaginal disk expression of decapentaplegic, a member of the TGF- $\beta$ family of Drosophila. Development 111: 657-665.

Bonifer, C., M. Vidal, F. Grosveld, and A.E. Sippel. 1990. Tissue specific and position independent expression of the complete gene domain for chicken lysozyme in transgenic mice. EMBO I. 9: 2843-2848.

Brook, W.J., L.M. Ostafichuk, J. Piorecky, M.D. Wilkinson, D.J. Hodgetts, and M.A. Russell. 1993. Gene expression during imaginal disc regeneration detected using enhancer-sensitive P-elements. Development 117: 1287-1297.

Cai, H. and M. Levine. 1995. Modulation of enhancer-promoter interactions by insulators in the Drosophila embryo. Nature 376: $533-536$.

Cherbas, L. and P. Cherbas. 1993. The arthropod initiator: The capsite consensus plays an important role in transcription. Insect Biochem. Mol. Biol. 23: 81-90.

Choi, O. and J.D. Engel. 1988. Developmental regulation of $\beta$-globin gene switching. Cell 55: 17-26.

Chung, J., M. Whiteley, and G. Felsenfeld. 1993. A 5 ' element of the chicken $\beta$-globin domain serves as an insulator in human erythroid cells and protects against position effect in Drosophila. Cell 74: 505-514.

Chung, Y.-T. and E.B. Keller. 1991. The TATA-dependent and TATA-independent promoters of the Drosophila melanogaster actin 5C-encoding gene. Gene 106: 237-241.
Corbin, V. and T. Maniatis. 1989. The role of specific enhancerpromoter interactions in the Drosophila Adh promoter switch. Genes \& Dev. 3: 2191-2201.

Corces, V. 1995. Keeping enhancers under control. Nature 376: $462-463$.

Das, G., C.S. Hinkley, and W. Herr. 1995. Basal promoter elements as a selective determinant of transcriptional activator function. Nature 374: 657-659.

Dascher, C., R. Ossig, D. Gallwitz, and H. Schmitt. 1991. Identification and structure of four yeast genes $(S L Y)$ that are able to suppress the functional loss of YPT1, a member of the RAS superfamily. Mol. Cell. Biol. 11: 872-885.

Dillon, N. and F. Grosveld. 1994. Chromatin domains as potential units of eukaryotic gene function. Curr. Opin. Genet. Dev. 4: 260-264.

Eissenberg, J.C. and S.C.R. Elgin. 1991. Boundary functions in the control of gene expression. Trends Genet. 7: 335-340.

Elgin, S.C.R. 1990. Chromatin structure and gene activity. Curr. Opin. Cell Biol. 2: 437-445.

Engels, W.R., D.M. Johnson-Schlitz, W.B. Eggleston, and J. Sved. 1990. High-frequency P element loss in Drosophila is homolog dependent. Cell 62: 515-525.

Ferguson, E.L. and K.V. Anderson. 1992. Decapentaplegic acts as a morphogen to organize dorsal/ventral pattern in the Drosophila embryo. Cell 71: 451-461.

Forrester, W.C., C. Thompson, J.T. Elder, and M. Groudine. 1986. A developmentally stable chromatin structure in the human $\beta$-globin gene cluster. Proc. Natl. Acad. Sci. 83: $1359-1363$.

Frohman, M.A., M.K. Dush, and G.R. Martin. 1988. Rapid production of full-length cDNAs from rare transcripts: Amplification using a single gene-specific oligonucleotide primer. Proc. Natl. Acad. Sci. 85: 8998-9004.

Gelbart, W.M. 1982. Synapsis-dependent allelic complementation at the decapentaplegic gene complex in Drosophila melanogaster. Proc. Natl. Acad. Sci. 79: 2636-2640.

Geyer, P.K. and V.G. Corces. 1987. Separate regulatory elements are responsible for the complex pattern of tissue-specific and developmental transcription of the yellow locus in Drosophila melanogaster. Genes \& Dev. 1: 996-1004.

- 1992. DNA position-specific repression of transcription by a Drosophila zinc finger protein. Genes \& Dev. 6: 18651873.

Gindhart, I.G., A.N. King, and T.C. Kaufman. 1995. Characterization of the cis-regulatory region of the Drosophila homeotic gene Sex combs reduced. Genetics 139: 781-795.

Gloor, G.B., N.A. Nassif, D.M. Johnson-Schlitz, C.P. Preston, and W.R. Engels. 1991. Targeted gene replacement in Drosophila via P-element induced gap repair. Science 253: 11101117.

Glover, D.M., M.H. Leibowitz, D.A. McLean, and H. Parry. 1995. Mutations in aurora prevent centrosome separation leading to the formation of monopolar spindles. Cell 81: 95105.

Grosveld, F., G. Blom van Assendelft, D.R. Greaves, and G. Kollias. 1987. Position-independent, high level expression of human $\beta$-globin gene in transgenic mice. Cell 51: 975-985.

Hansen, S.K. and R. Tjian. 1995. TAFs and TFIIA mediate differential utilization of the tandem $A d h$ promoters. Cell 82: $565-575$.

Holdridge, C. and D. Dorsett. 1991. Repression of hsp 70 heat shock gene transcription by the suppressor of Hairy-wing protein of Drosophila melanogaster. Mol. Cell. Biol. 11: 1894-1900.

Ingham, P.W. and M.J. Fietz. 1995. Quantitative effects of hedgehog and decapentaplegic activity on the patterning of 
the Drosophila wing. Curr. Biol. 5: 432-440.

Kania, A., A. Salzberg, M. Bhat, D. D'Evelyn, Y. He, I. Kiss, and H.J. Bellen. 1995. P-element mutations affecting embryonic peripheral nervous system development in Drosophila melanogaster. Genetics 139: 1663-1678.

Kapoun, A.M. and T.C. Kaufman. 1995. A functional analysis of $5 '$, intronic and promoter regions of the homeotic gene proboscipedia in Drosophila melanogaster. Development 121: 2127-2141.

Karess, R.E. and G.M. Rubin. 1984. Analysis of P transposable element functions in Drosophila. Cell 38: 135-146.

Kellum, R. and P. Schedl. 1991. A position-effect assay for boundaries of higher order chromosomal domains. Cell 64: 941-950.

1992. A group of scs elements function as domain boundaries in an enhancer-blocking assay. Mol. Cell. Biol. 12: 2424-2431.

Kitsberg, D., S. Selig, and H. Cedar. 1991. Chromosome structure and eucaryotic gene organization. Curr. Op. Gen. Dev. 1: 534-537.

Knipple, D.C. and P. Marsella-Herrick. 1988. Versatile plasmid vectors for the construction, analysis and heat-inducible expression of hybrid genes in eukaryotic cells. Nucleic Acids Res. 16: 7748.

Laemmli, U.K., E. Käs, L. Poljak, and Y. Adachi. 1992. Scaffoldassociated regions: Cis-acting determinants of chromatin structural loops and functional domains. Curr. Opin. Genet. Dev. 2: 275-285.

Lee, H.-S., K.W. Kraus, M.F. Wolfner, and J.T. Lis. 1992. DNA sequence requirements for generating paused polymerase at the start of hsp70. Genes \& Dev. 6: 284-295.

Lewin, B. 1994. Chromatin and gene expression: Constant questions, but changing answers. Cell 79: 397-406.

Li, X. and M. Noll. 1994. Compatibility between enhancers and promoters determines the transcriptional specificity of gooseberry and gooseberry neuro in the Drosophila embryo. EMBO /. 13: 400-406.

Lindsley, D.L. and G.G. Zimm. 1992. The genome of Drosophila melanogaster. Academic Press, San Diego, CA.

Lis, J. and C. Wu. 1993. Protein traffic on the heat shock promoter: Parking, stalling, and trucking along. Cell 74: $1-4$.

Littleton, T. and H. Bellen. 1994. Genetic and phenotypic analysis of 13 essential genes in the cytological interval 22F1-2; 23B1-2 reveals three genes required for neural development in Drosophila. Genetics 138: 111-123.

Martinez, E., C.-M. Chiang, H. Ge, and R.G. Roeder. 1994. TATA-binding protein-associated factor(s) in TFIID function through the initiator to direct basal transcription from a TATA-less class II promoter. EMBO I. 13: 3115-3126.

Masucci, J.D., R.J. Miltenberger, and F.M. Hoffmann. 1990. Pattern-specific expression of the Drosophila decapentaplegic gene in imaginal disks is regulated by $3^{\prime}$ cis-regulatory elements. Genes \& Dev. 4: 2011-2023.

McKnight, R., A. Shamay, L. Sankaran, R. Wall, and L. Hennighausen. 1992. Matrix-attachment regions can impart position-independent regulation of a tissue-specific gene in transgenic mice. Proc. Natl. Acad. Sci. 89: 6943-6947.

Mizuta, K. and J. Warner. 1994. Continued functioning of the secretory pathway is essential for ribosome synthesis. Mol. Cell. Biol. 14: 2493-2502.

Nassif, N., J. Penny, S. Pal, W.R. Engels, and G.B. Gloor. 1994. Efficient copying of nonhomologous sequences from ectopic sites via P-element-induced gap repair. Mol. Cell. Biol. 14: 1613-1625.

Ossig, R., C. Dascher, H. Trepte, H. Dieter, and D. Gallwitz. 1991. The yeast SLY gene products, suppressors of defects in the essential GTP-binding Yptl protein, may act in endoplasmic reticulum-to-golgi transport. Mol. Cell. Biol. 11: 2980-2993.

Parvin, J.D., H.T.M. Timmers, and P.A. Sharp. 1992. Promoter specificity of basal transcription factors. Cell 68: 1135-1144.

Ponce, M.R. and J.L. Micol. 1992. PCR amplification of long DNA fragments. Nucleic Acids Res. 20: 623.

Raftery, L.A., M. Sanicola, R.K. Blackman, and W.M. Gelbart. 1991. The relationship of decapentaplegic and engrailed expression in Drosophila imaginal disks: Do these genes mark the anterior-posterior compartment boundary? Development 113: 27-33.

Robertson, H.M., C.R. Preston, R.W. Phillis, D.M. JohnsonSchlitz, W.K. Benz, and W.R. Engels. 1988. A stable source of $P$ element transposase in Drosophila melanogaster. Genetics 118: 461-470.

Roseman, R.R., V. Pirrotta, and P.K. Geyer. 1993. The su(Hw) protein insulates expression of the Drosophila melanogaster white gene from chromosomal position-effects. EMBO $/$. 12: $435-442$.

Salzberg, A., N. Cohen, N. Halachmi, Z. Kimchie, and Z. Lev. 1993. The Drosophila Ras2 and Rop gene pair: A dual homology with a yeast Ras-like gene and a suppressor of its loss-of-function phenotype. Development 117: 1309-1319.

Schedl, P. and F. Grosveld. 1995. Domains and boundaries. In Chromatin structure and gene expression (ed. S.C.R. Elgin), pp. 172-196. IRL Press, Oxford, UK.

Scott, K.S. and P.K. Geyer. 1995. Effects of the su(HW) insulator protein on the expression of the divergently transcribed Drosophila yolk protein genes. EMBO J. 14: 6258-6267.

Spencer, F.A., F.M. Hoffmann, and W.M. Gelbart. 1982. Decapentaplegic: A gene complex affecting morphogenesis in Drosophila melanogaster. Cell 28: 451-461.

St. Johnston, R.D. and W.M. Gelbart. 1987. Decapentaplegic transcripts are localized along the dorsal-ventral axis of the Drosophila embryo. EMBO I. 6: 2785-2791.

St. Johnston, R.D., F.M. Hoffmann, R.K. Blackman, D. Segal, R. Grimaila, R.W. Padgett, H.A. Irick, and W.M. Gelbart. 1990. Molecular organization of the decapentaplegic gene in Drosophila melanogaster. Genes \& Dev. 4: 1114-1127.

Stief, A., D.M. Winter, W.H. Strätling, and A. E. Sippel. 1989. A nuclear DNA attachment element mediates elevated and position-independent gene activity. Nature 341: 343-345.

Vasquez, J. and P. Schedl. 1994. Sequences required for enhancer blocking activity of scs are located within two nucleasehypersensitive regions. EMBO I. 13: 5984-5993.

Verrijzer, C., J. Chen, K. Yokomori, and R. Tjian. 1995. Binding of TAFs to core elements directs promoter selectivity by RNA polymerase II. Cell 81: 1115-1125.

Wefald, F.C., B.H. Devlin, and R.S. Williams. 1990. Functional heterogeneity of mammalian TATA-box sequences revealed by interaction with a cell-specific enhancer. Nature 344: 260-262.

Wharton, K.A., R.P. Ray, and W.M. Gelbart. 1993. An activity gradient of decapentaplegic is necessary for the specification of dorsal pattern elements in the Drosophila embryo. Development 117: 807-822.

Wiigerde, M., F. Grosveld, and P. Fraser. 1995. Transcription complex stability and chromatin dynamics in vivo. Nature 377: 209-213.

Wingender, E., P. Dietze, H. Karas, and R. Knüppel. 1996. TRANSFAC: A database on transcription factors and their DNA binding sites. Nucleic Acids Res. 24: 238-241.

Zecca, M., K. Basler, and G. Struhl. 1995. Sequential organizing activities of engrailed, hedgehog, and decapentaplegic in the Drosophila wing. Development 121: 2265-2278. 


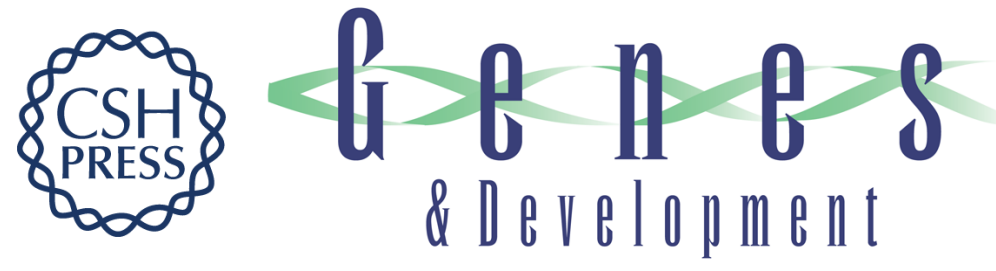

\section{Promoter specificity mediates the independent regulation of neighboring genes.}

C Merli, D E Bergstrom, J A Cygan, et al.

Genes Dev. 1996, 10:

Access the most recent version at doi:10.1101/gad.10.10.1260

References This article cites 70 articles, 30 of which can be accessed free at:

http://genesdev.cshlp.org/content/10/10/1260.full.html\#ref-list-1

License

Email Alerting

Service

Receive free email alerts when new articles cite this article - sign up in the box at the top right corner of the article or click here. 